\title{
Who's for growth hormone?
}

\section{Growth hormone should be given only to short children with demonstrably inadequate secretion of the natural hormone}

When growth hormone was extracted from human pituitary glands its use was restricted to short children growing slowly with a demonstrable insufficiency of growth hormone secretion. The asymptotic shape of the relation between growth hormone secretion and the rate at which a child grows ${ }^{1}$ indicates just how cost effective was that selection. The problem is that there is no cut off that defines growth hormone insufficiency and no child who will not respond by growing more quickly if given a sufficient dose of growth hormone. If there is an unlimited supply of the stuff who should get it?

The response of a child to growth hormone is defined by the pretreatment growth velocity (which reflects the endogenous growth hormone secretory capacity), the dose of growth hormone used, the frequency of administration, and the condition being treated. ${ }^{2}$ It is in the quest to define the scope of the last variable that many trials of growth hormone have been initiated-including conditions as diverse as chronic renal failure, skeletal dysplasias, Turner's syndrome, Noonan's syndrome, intrauterine growth retardation, and normal children, to name but a few. Interest now centres on even wider indications such as "the syndrome of GH insufficiency" in adults ${ }^{34}$ and the correction of catabolic states after extensive burns or surgery. ${ }^{56}$

Apart from elderly patients, in whom it may provoke water retention, growth hormone has been remarkably free from side effects over the 30 years in which it has been in clinical use. The contamination of the pituitary derived product with the causative agent of Creuzfeldt-Jacob disease is not the fault of growth hormone. There are dangers of using an agent that is designed to increase cell division, but the risk of inducing neoplastic change seems to be extraordinarily small. There are theoretical dangers of using a material that increases free fatty acid and insulin concentration and causes water retention (which possibly increases blood pressure), which might accelerate the process of atherosclerosis, but in fact patients with hypopituitarism have excess mortality from this cause. ${ }^{7}$

If growth hormone increases the growth rate of any child to whom it is given why should it not be given to all short children? After all, tall people in general and pituitary giants in particular become tall only by growing consistently at a rate faster than they would otherwise be expected to grow. The straight answer to the question is that giving growth hormone to children other than those with growth hormone insufficiency seems rarely to achieve a consistent increase in growth rate year after year, and it may well shorten the duration of the whole growing period. Such an effect becomes particularly noticeable at puberty, ${ }^{8}$ but is also seen in younger children. For example, children with intrauterine growth retardation can be made to grow more quickly by giving them growth hormone but predictions of their final height could not be altered by this treatment. ${ }^{9}$ As what matters is the final height achieved, guidelines on who should be treated with growth hormone are fairly easily formulated. Such selection is important because growth hormone is expensive. The Department of Health is happy to see a pattern of shared care emerging between hospital specialists and family practitioners to spread the cost of prescribing, ${ }^{10}$ but the details of the product licence should be observed.

Short children should be identified by primary care physicians. Their rate of growth should be assessed over not less than six months and not more than one year. Criteria for deciding when to intervene and investigate have been discussed in detail previously. "Briefly, a child who grows less than $4 \mathrm{~cm}$ in any one year requires an explanation; a child growing between this rate and the 25 th centile velocity for age for two years also requires investigation, diagnosis, and treatment. Short children growing slowly should be referred for investigation. When other causes of failing to thrive have been excluded, usually by clinical examination, an endocrine cause may be considered. Insufficiency of growth hormone secretion is the commonest of such causes. The results of tests of growth hormone secretion are difficult to interpret ${ }^{12}$ and commonly used tests may be dangerous in inexperienced hands ( $p$ 173). ${ }^{13}$ The very few children who really need these tests should therefore be investigated in centres for growth assessment that are properly staffed and equipped to investigate hormone secretion efficiently, effectively, safely, and with knowledge of the limitations of the results of the test.

When such a centre has diagnosed growth hormone insufficiency the child should be sent back to the referring physician, who should be told the recommended dose and mode of administration. A wise physician will from time to time be glad to have the child reviewed in the centre, but treatment should be managed locally.

Finally, there is no place for the one off prescription of growth hormone to any child. Until the current carefully constituted trials of growth hormone in conditions other than growth hormone insufficiency have reported their findings to 
final height (and that includes in my view patients with Turner's syndrome) children in these categories should be treated only within the confines of a clinical trial. ${ }^{14}$ From recent experience it seems that giving growth hormone to normal children may result in an increase in final stature of about $4 \mathrm{~cm}$ after 10 years of treatment at a cost of $£ 80000$ and the trauma of daily injections. ${ }^{15}$ This hardly seems warranted.

A possible way forward in several groups of short children, particularly short normal ones and girls with Turner's syndrome, may be to induce a short term increase in growth rate in childhood to alleviate the handicap that short stature in childhood undoubtedly constitutes. The effect of a short (one year) course of growth hormone for candidate groups of short children is probably the next clinical question to answer.

C G D BROOK

Professor of Paediatric Endocrinology,

Middlesex Hospital,

London W1N 8AA
1 Hindmarsh PC, Smith PJ, Brook CGD, Matthews DR. The relationship between height velocity and growth hormone secretion in short prepubertal children. Clin Endocrinol 1987;27:581-91. 2 Darendeliler F, Hindmarsh PC, Brook CGD. Dose response curves for treatment with biosynthetic human growth hormone. $\mathcal{f}$ Endocrinol 1990;125:311-6.

3 Salomon F, Cuneo RC, Hesp R, Sönksen PH. The effects of treatment with recombinant human growth hormone on body composition and metabolism in adults with growth hormon deficiency. $N$ Engl f Med 1989;321:1797-803.

4 Rudman DR, Feller AG, Hagraj HS, Gergans GA, Lalitha PY, Goldberg RA, et al. Effects of human growth hormone in men over 60 years old. $N$ Engl f Med 1990;323:1-6.

Wilmore DW. Catabolic illness strategies for enhancing recovery. N Engl f Med 1991;325:695-702. 6 Ross RJM, Miell JP, Buchanan CR. Avoiding autocannibalism. BMF1991;303:1147-8.

Rosén T, Bengtsson B-A. Premature mortality due to cardiovascular disease in hypopituitarism. Lancet 1990;336:285-8.

8 Darendeliler F, Hindmarsh PC, Preece MA, Cox L, Brook CGD. Growth hormone increases rate of pubertal maturation. Acta Endocrinol 1990;120:414-6.

9 Stanhope R, Preece MA, Hamill G. Does growth hormone treatment improve final height attainment of children with intrauterine growth retardation? Arch Dis Child 1991;66:1180-3.

10 Walford D. Responsibility for prescribing between hospitals and GPs. London: NHS Management Executive, 1991. (EL(91)127.)

11 Brook CGD, Hindmarsh PC, Healy MJR. A better way to detect growth failure. BMF 1986;293:1186.

12 Brook CGD, Hindmarsh PC. Tests for growth hormone secretion. Arch Dis Child 1991;66:85-7. 13 Shah A, Stanhope R, Matthew D. Hazards of pharmacological tests of growth hormone secretion in childhood. BMF 1992;304:173-4.

14 Hindmarsh PC, Bridges NA, Brook CGD. Wider indications for treatment with biosynthetic human growth hormone in children. Clin Endocrinol 1991;34:417-27.

15 Albertsson-Wikland K, Karlberg J. Effect of GH treatment in short children on the timing of puberty and final height. Horm Res 1991;35(suppl 2):32.

\title{
Monoclonal antibodies in sepsis and septic shock
}

\author{
Recent progress, vast potential
}

Attempts to combat the high mortality from sepsis initially concentrated on patients who were shocked and led to an appreciation that prompt cardiorespiratory resuscitation, including mechanical ventilation, could substantially improve outcome. ${ }^{1}$ Efforts to reduce mortality further by inhibiting inflammatory mediators have so far proved disappointing, but hope now exists that monoclonal antibodies, which can be precisely targeted against individual mediators or their receptors, may allow more effective manipulation of the systemic response to infection.

Monoclonal antibodies have been raised against tumour necrosis factor, a cytokine central to the initiation of the inflammatory response, and against tumour necrosis factor receptors. ${ }^{2}$ Administering antibodies to tumour necrosis factor has produced encouraging results in animal models of septic shock, ${ }^{34}$ and its safety has been shown in a small phase 1 clinical study. ${ }^{5}$ Cytokines, however, play an important part in host resistance, and inhibition could impair patients' ability to eradicate infection and increase their susceptibility to secondary sepsis. Conversely, inhibiting only one of the many cytokines involved in the response to infection may not be sufficient.

Targeting the toxic products of the infecting organism rather than the endogenous mediators of the inflammatory process might overcome these problems. Directing treatment specifically against circulating endotoxin (the lipopolysaccharide component of the bacterial cell wall that is responsible for many of the systemic responses to Gram negative infection) is now possible by raising antibodies to the structurally conserved core glycolipid of endotoxin, or against lipid A itself, rather than the very immunogenic but variable oligosaccharide side chains. Compared with treatments directed against inflammatory mediators this approach is obviously limited to cases of Gram negative bacteraemia or endotoxaemia.

Encouraging results have been obtained with human polyclonal antiserum directed against endotoxin core determinants, ${ }^{6-8}$ and the importance of the IgM class of immunoglobulins in providing this protection is now appreciated..$^{910}$
The practical difficulties associated with using polyclonal antiserum have recently been circumvented with monoclonal antibodies such as HA-1A, a human monoclonal IgM antibody that binds specifically to many endotoxins as well as to a broad range of clinical isolates of Gram negative bacteria. After studies showing its efficacy in animal models ${ }^{112}$ and initial evaluations in humans ${ }^{1314}$ a rigorous multicentre, prospective, randomised controlled trial of HA-1A has recently been completed. ${ }^{15}$ Five hundred and forty three patients suspected of having Gram negative infection and fulfilling predefined criteria for sepsis were enrolled; in the 200 who were subsequently proved to have had Gram negative bacteraemia HA-1A significantly reduced 28 day mortality by an impressive $39 \%$ in the 105 patients receiving the drug and significantly increased the number of patients who were discharged from hospital.

These days, however, even the most impeccably designed study inevitably arouses criticism and comment. Concern has been expressed that the mortality of all 543 patients enrolled in the study was not significantly different between the two groups (HA-1A 39\%; placebo 43\%) and that in those without Gram negative bacteraemia mortality was higher in those given HA-1A (45\%) than in the controls (40\%) (although this difference was not significant). Others have been concerned by uncertainties about the mechanism of protection by both polyclonal antiserum ${ }^{1617}$ and monoclonal antiendotoxin antibodies, ${ }^{18}{ }^{19}$ and the specificity of HA-1A for lipid A has been questioned. $^{19}$

The lack of efficacy of HA-1A in the 201 patients with Gram negative infection but without bacteraemia is difficult to explain as endotoxaemia often occurs without bacteraemia. ${ }^{20}$ This also contrasts with the observation that E5, a murine IgM antiendotoxin monoclonal antibody, improved outcome in non-bacteraemic as well as bacteraemic patients with Gram negative infection. ${ }^{21}$ It is also disconcerting that, in contrast to HA-1A, which was effective irrespective of whether or not the patient was shocked, ${ }^{21} \mathrm{E} 5$ was ineffective in those with "refractory" shock. These discrepancies may, of course, be due to differences between human and murine 